\title{
Changes in behavior of hippocampal-lesioned rats across a 6-week postoperative period
}

\author{
DANIEL PORTER KIMBLE \\ University of Oregon, Eugene, Oregon 97403
}

\begin{abstract}
The postoperative behavior of rats with one-stage bilateral lesions of the dorso-lateral hippocampus was examined preoperatively and on 15 separate occasions over a 42-day postoperative period. Repeated measures of spontaneous alternation in a T-maze and general activity in an open field were made. The postoperative behavior of the hippocampal-lesioned rats was compared with that of control rats which had sustained one-stage bilateral lesions of the neopallium overlying the hippocampal formation. The effect of the neopallial lesion on both behaviors was transitory; preoperative levels of behavior returned within $72 \mathrm{~h}$ postoperatively. For the hippocampal-lesioned group there was a drop in the level of spontaneous alternation on Day 2 postoperatively, a brief return to preoperative levels on Day 3, and then a sustained depression in rate until Day 14, when a return toward preoperative alternation rates occurred. In the open field, the hippocampal-lesioned subjects displayed a 3-4-day period of hypoactivity, followed abruptly by a 7-9-day period of hyperactivity. These subjects then returned to preoperative levels of activity. The nature and time course of these shifts in postoperative behavior were discussed in terms of their possible relationship to findings regarding synaptic reinnervation of the septal nuclei.
\end{abstract}

A large number of investigators have reported on the behavioral effects of hippocampal lesions, but few have examined in detail the time course of behavioral changes following surgery. Typically, experiments in this area have been concerned with the acquisition of some learned task at a given "recovery" period following surgery. These postoperative "recovery" periods have been as short as 2 days (Lanier \& Isaacson, 1975) to a month or longer (Kimble \& Kimble, 1970; Roberts, Dember, \& Brodwick, 1962). One study has been reported which does address itself to postoperative differences as a function of time after surgery (Dawson, Conrad, \& Lynch, 1975). However, due to the nature of the experimental design used in that study, repeated measures across the postoperative period were not done. The data reported in the present paper are relevant to a consideration of the possible changes in behavior seen during a 6-week postoperative period following one-stage bilateral lesions. These data enlarge the store of knowledge regarding the behavioral effects of hippocampal lesions and support the concept of the first few weeks of the postoperative period as one of behavioral change.

\section{METHOD}

\section{Animals}

The subjects were male albino rats derived from a strain obtained from Carworth Farms and bred in the Oregon Psychology Department Animal Laboratory. The rats were from three litters born within a 10-day period; they were between 100 and 110 days of age at the beginning of postoperative behavioral testing. The animals were divided into two groups of five on the basis of their preoperative open-field and spontaneous alternation be-

I would like to thank Ruth BreMiller for the histology. havior. One group of five then received bilateral lesions to the dorsolateral aspect of the hippocampal formation. The other group received bilateral lesions to the neopallium overlying the hippocampus. One of the rats assigned to the neopallial-lesion group died after receiving Nembutal preparatory to surgery. All other rats survived and finished the entire experiment.

\section{Surgery and Histology}

All operations were performed under clean conditions. The lesions were performed by aspiration under visual control, using a dissecting microscope. The anesthetic agent used was Nembutal $(50 \mathrm{mg} / \mathrm{kg})$. The lesions were made in one stage, following a procedure reported previously (Kimble, 1963). Each rat received an IM injection of a broad-spectrum antibiotic within $2 \mathrm{~h}$ after surgery. No infection or illness occurred in any animal throughout the duration of the experiment. All rats were sacrificed at 60-65 days postoperatively, the brains removed, frozen, sectioned at 12 microns, and stained with thionin according to a procedure developed in our laboratory (BreMiller, 1971).

\section{Apparatus}

For examining spontaneous alternation, the apparatus used was a wooden T-maze. The stem of the maze was $56.3 \mathrm{~cm}$ long $\mathrm{x}$ $11.4 \mathrm{~cm}$ wide. This was at tached to a $25.4 \times 11.4 \mathrm{~cm}$ start box. Each goal arm was $31.7 \mathrm{~cm}$ long $x 24.1 \mathrm{~cm}$ wide. A $21.6-\mathrm{cm}$ partition partially divided each goal arm. The apparatus was $17.8 \mathrm{~cm}$ high. A removable sheet of Plexiglas served as the floor of the maze, and four hinged Plexiglas panels formed tops for the startbox, main stem, and two goal arms. Plexiglas guillotine doors separated the main stem from the goal arms and were manipulated manually by the experimenter.The wooden partitions of the maze were painted a medium flat gray.

For observation of the open-field behavior, a $78.7 \times 78.7 \mathrm{~cm}$ box was used. The walls were $11.4 \mathrm{~cm}$ high. A sheet of clear Plexiglas served as a cover. The apparatus was painted a medium flat gray, and the floor of the field was marked off into 36 equalsized squares by white painted lines.

\section{Procedure}

The animals were housed individually and maintained on an 


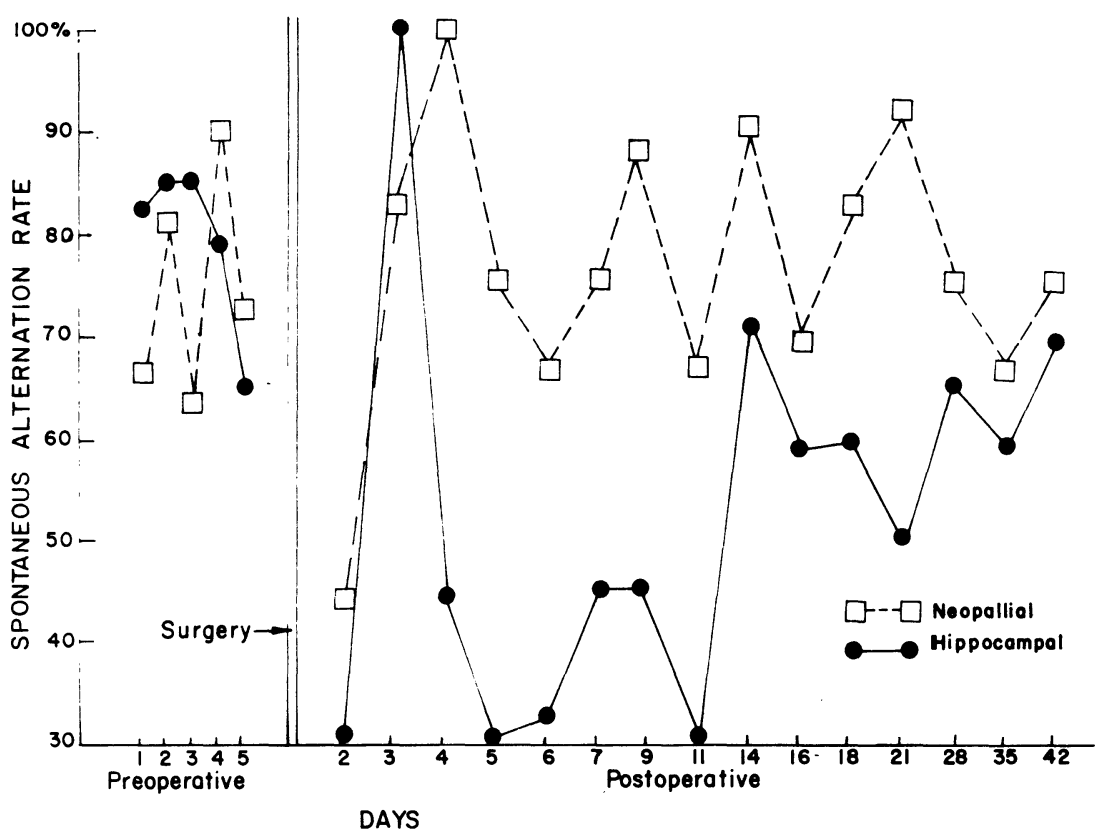

Figure 1. Spontaneous alternation, preand postoperative, hippocampal-lesion and neopallial-tesion groups. Curves represent mean alternation scores for that day.

ad-lib food and water schedule. The room in which they were housed and tested was placed on a 12-12-h light-dark schedule, with the light cycle beginning at 6:00 a.m. Testing was done in the middle of the light cycle. A "follow-up" testing session was run during the middle of the dark cycle 1 week after completion of the light-cycle testing. Although all of the animals were initially quite gentle, additional gentling was accomplished by handling each rat for approximately $5 \mathrm{~min}$ each day for 5 days prior to the beginning of the preoperative testing.

Preoperative testing. Prior to surgery, each rat was tested for 5 consecutive days for both spontaneous alternation and openfield activity. The spontaneous alternation tests took place in the wooden T-maze described above. No food or water was ever present in the maze. The Plexiglas floor of the T-maze was removed, washed with warm water, dried with paper toweling, and replaced between each rat's daily test. Each subject was given five spontaneous alternation trials daily. A trial was initiated by placing the rat in the startbox and lifting the guillotine door separating the startbox from the main stem, as well as those separating the main stem from the goal arms. After the rat entered one of the goal arms, the guillotine doors were gently lowered, and 20 sec later the rat was removed and replaced in the startbox for the next trial. If a subject refused to enter (all four limbs within a goal arm) within $3 \mathrm{~min}$, that trial was terminated and a new trial started. Each rat was given five trials each day. Trials 2-5 were the "test" trials, and an animal could thus score a maximum of four alternations per day. At the end of its fifth trial, each subject was returned to its home cage. When all the rats had been tested for spontaneous alternation, they were examined in the open-field apparatus.

Following the spontaneous alternation tests, each subject was placed, individually, in the center of the open field, and the Plexiglas cover was set in place. The number of two-footed entries into the floor squares was recorded each minute for $10 \mathrm{~min}$, along with the number of fecal boluses and urinations, as well as other behaviors, such as rearing, grooming, etc. After each subject's testing period, the floor of the field was washed with warm water and dried with paper toweling.

Postoperative testing. All subjects were examined for both spontaneous alternation levels and open-field activity on each of the following postoperative days: $2,3,4,5,6,7,9,11,14$, $16,18,21,28,35$, and 42 . Thus, there were 5 days of preoperative testing and 15 days postoperatively. The procedures for the postoperative period were identical with those already described for the preoperative period.

\section{RESULTS}

\section{Spontaneous Alternation}

All subjects showed typical spontaneous alternation behavior preoperatively. The mean alternation rate for the neopallial-lesioned control rats was $75 \%$, while that for the hippocampal-lesioned group was $83 \%$. Postoperatively, there was a drop in the alternation rate for both groups on the first test day following surgery (Day 2, $48 \mathrm{~h}$ after surgery). On Postoperative Day 3, the spontaneous alternation rates for those animals who completed three or more test trials (2/4 neopalliallesion and $2 / 5$ hippocampal-lesion animals) was at or above preoperative alternation levels. By Postoperative Day 4, all subjects completed two or more trials. On this day, a sharp difference in spontaneous alternation levels appeared, with the neopallial-lesion group alternating at the $100 \%$ level (16 alternations out of 16 trials), while the hippocampal-lesion group fell to an alternation rate of $44 \%$ ( 7 alternations out of 16 trials). From Day 4 through the duration of the experiment, a difference in alternation rate persisted. The neopallial-lesion group continued to show spontaneous alternation rates that averaged $77 \%$ across the 6 -week postoperative period, while the hippocampal-lesion group averaged 53\% across the postoperative period. Although the spontaneous alternation rate of the hippocampal-lesion group never returned to preoperative levels, from Day 14 onward there was a clear increase in the alternation rate, and by Day 42 there was only a $6 \%$ difference in the alternation rate between the two groups. By this time, $3 / 5$ hippocampal-lesion subjects had displayed 2 or more consecutive days of $75 \%$ alternation or better. Animal 6-H (hippocampal-lesion), which had shown alternation rates of $50 \%$ or less for 11 consecutive testing sessions, alternated at the $100 \%$ level on Days 35 and 42 (8/8 trials). Figure 1 illustrates the 


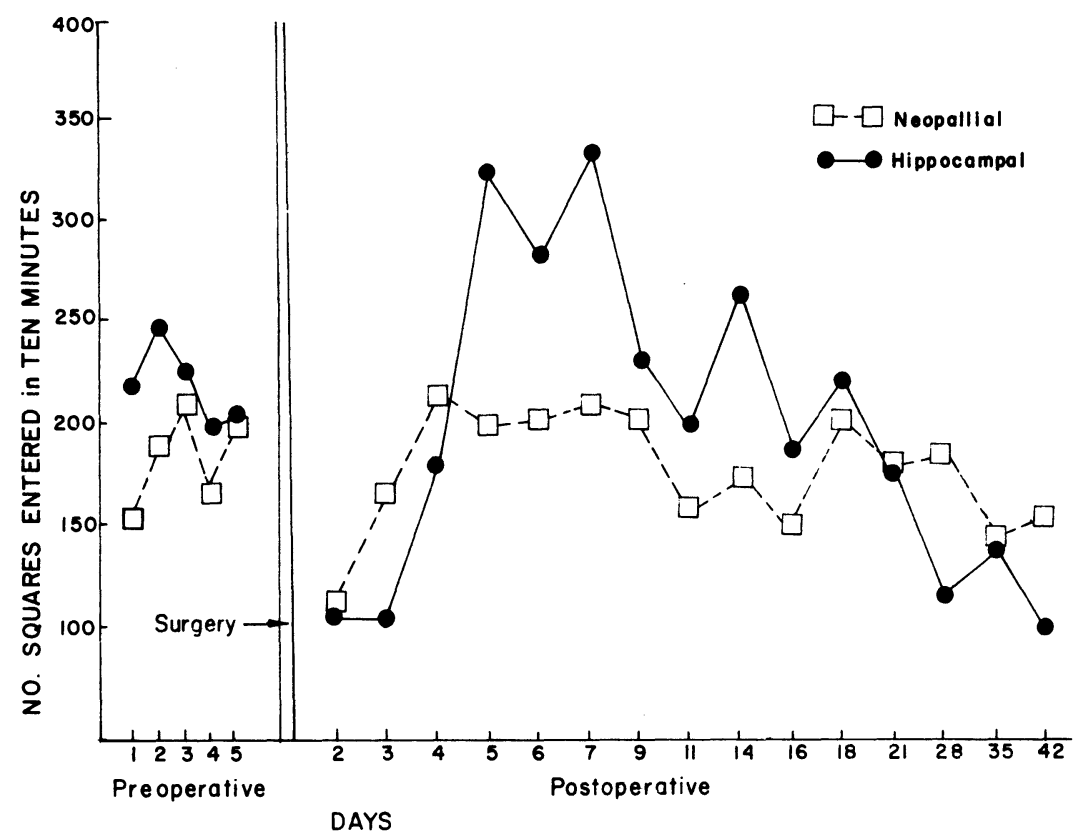

Figure 2. Number of squares entered in open field, pre- and postoperative, hippocampaltesion and neopallial-tesion groups. Curves represent mean entry scores for that day. group curves. An analysis of variance for repeated measures showed the differences between the two groups to be statistically significant $(F=6.21, p=.05)$. In summary, the effect of the neopallial-lesion on spontaneous alternation appears to be transitory, with complete recovery occurring by $72 \mathrm{~h}$ postoperatively. The rate of alternation in this group was then indistinguishable from the preoperative rate for the duration of the experiment. For the hippocampal-lesion group, there is also a depression on Day 2, a brief return to a high rate on Day 3, and then a depression in alternation rate until Day 14, where an elevation in rate was observed.

\section{Open Field Activity}

The experimental groups were constructed on the basis of their preoperative behavior. The death of one subject in the neopallial-lesion group left the two groups slightly (although insignificantly) different in preoperative open-field activity levels. The group assigned to the neopallial-lesion group had a mean preoperative activity score of 182 squares/10-min observation period, and the hippocampal-lesion group had a mean preoperative activity score of 218 squares/10-min observation period. Following surgery, all subjects in both groups showed lowered activity levels on Day 2. On Day 3, the neopallial-lesioned subjects had returned to preoperative activity levels, which were maintained for the duration of the experiment. The depressed activity levels among the hippocampal-lesioned subjects continued for 1 more day, returning to near-normal levels in $4 / 5$ subjects by Day 4. On Day 5, there was an abrupt increase in the activity levels among the hippocampal-lesion subjects, with all rats in this group exceeding their mean preoperative scores. This hyperactive period lasted until Days 11-14. By Day 16, activity levels had returned to normal, where they remained for the duration of the study. The hyperactivity displayed by the hippocampallesion rats during the period from Day 5 to Day 11 was very similar to that reported several years ago (Kimble, 1963). The rats, during this time, would run rapidly along the perimeter of the open field in an automaton fashion, only penetrating to the interior of the field after the first 5-6 min. During this period, it was not unusual for a rat to enter over 100 squares/min for 2-3 consecutive minutes, five times their average preoperative rate. It is relevant to note that the hyperactivity reported several years ago was evaluated on the 8th day following surgery (Kimble, 1963). Figure 2 shows the activity data.

During the brief period of hypoactivity preceding the hyperactive period, observation of the hippocampallesioned subjects revealed that there was a qualitative difference in their open-field behavior as compared with that of the neopallial-lesioned subjects. While the neopallial-lesioned subjects were less active on Day 2, their locomotion appeared to be normal. The behavior of the hippocampal-lesioned subjects, on the other hand, appeared quite abnormal. When placed in the open field (or in the T-maze), 4/5 hippocampal-lesioned subjects displayed an extreme "lethargy" in their movements. They tended to retain a given posture for long periods of time, and when they did move, they did so in a "slowmotion," slinking fashion. Also, the exactness with which these subjects would retain a particular posture was remarkable; they often held a pose unchanged for several minutes at a time. This inertia gave way overnight between the 4th and 5th day postoperatively for the $4 / 5$ subjects in which it appeared. This phenomenon was never observed in the neopallial-lesioned subjects.

One week following the Day 42 tests; all subjects were examined for activity during the middle of their dark cycle. Although activity levels were approximately 


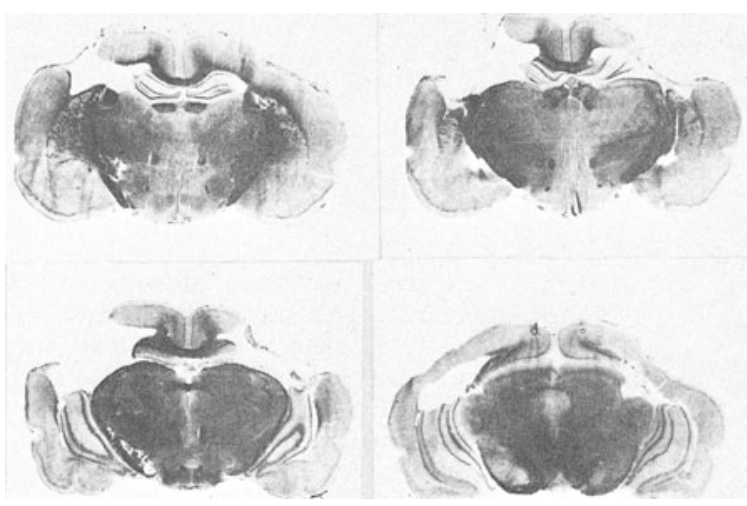

Figure 3. Frontal sections from brain of representative hippocampal-tesion rat.

$30 \%$ higher for all subjects, no significant differences between the two groups of animals were seen. An analysis of variance performed on the light-cycle activity data was not significant, as the hippocampal-lesion periods of hypoactivity and hyperactivity apparently tended to cancel each other out statistically $(F=.94)$.

\section{Histology}

In the hippocampal-lesioned subjects, the surgery resulted in substantial-to-complete ablation of dorsal and dorsolateral portions of the hippocampus and dentate gyrus, including the dorsal fornix and portions of the subiculum. Some invasion of the entorhinal cortex was observed in all subjects, but the extent was quite minimal. The degree of sparing of the ventral hippocampal formation was somewhat more variable. The brain sections pictured in Figure 3 are from a representative hippocampal-lesioned rat. In two of the five subjects, some slight inadvertant damage was present unilaterally in the dorso-lateral thalamus. It was primarily located in the area of the nucleus suprageniculatus and pretectal nucleus and pretectal nucleus profundus.

The brains of the rats with neopallial lesions presented a uniform picture. The neopallium overlying the dorsal and lateral aspects of the hippocampal formation was removed, as illustrated in Figure 4. The total amount of brain tissue removed in the two groups was nearly equal, as the neopallial lesion was extended to incorporate more tissue than was the case for the neopallial lesion in the hippocampal-lesioned subjects.

\section{DISCUSSION}

The 6-week postoperative period examined in this study revealed that behavioral changes that can be reliably found after 1-2 weeks following hippocampal lesions in rats may be different or vanished by 3-4 weeks. It is, of course, possible that further behavioral changes might have been detected with an extended period of data collection. However, there are some reasons to believe that the initial 6 weeks following surgical intervention in the adult rat brain may be the period of greatest change. This period coincides with the time in which several investigators have discovered a substantial degree of "reinnervation" of regions of the brain which have been partially denervated by brain lesions (Moore, Bjorklund, \& Stenevi, 1971, 1974; Raisman, 1969; Raisman \& Field, 1973). In particular, this reinnervation of vacated synaptic sites has been reported in the septal area, following lesions to either the ascending limb of the medial forebrain bundle or the fimbria. Raisman's analysis of electron micrograph data (Raisman, 1969) led him to conclude that the reinnervation of the vacated synaptic sites in the septal area was due to the "filling in" or "reclamation" of these sites by collateral sprouting from the axons of remaining intact afferent fibers. Thus, following lesions to the fimbria, synaptic sites in both medial and lateral septal nuclei are apparently reoccupied by collateral sprouting from axons of the ascending medial forebrain bundle. Moore et al. $(1971,1974)$ have presented data which support this conclusion.

The time course for this "reclamation" by intact fibers is not as yet precisely known, but Moore et al. (1971) report that the first indications of increased septal innervation appear between 8 and 15 days following a hippocampal-fimbria lesion, that this reinnervation is quite marked by 30-60 days, and that it persists for at least 100 days. Similarly, Raisman has reported that by 42 days following fimbrial section, very few degenerating terminals can still be found in the septum and that "the general configuration of synapses has reached a stable state" (Raisman, 1969). Thus, it appears that, following surgical intervention similar to that performed in this study, the synaptic rearrangements in the septal area set in rapidly after surgery, are clearly noticeable within 2 weeks, and are far advanced and perhaps at a stable level by 6 weeks. It is, of course, tempting to speculate that the behavioral changes seen following hippocampal lesions are related in some meaningful
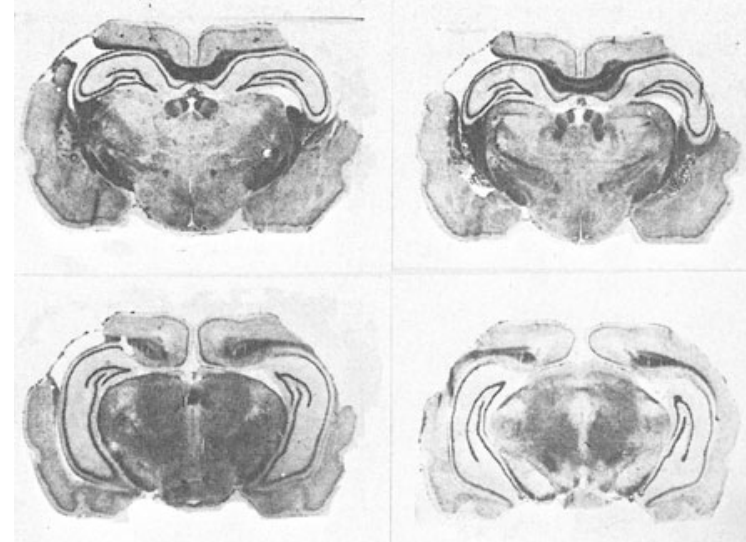

Figure 4. Frontal sections from brain of representative neopallial-tesion rat. 
way to these apparent synaptic changes, as observed by Raisman and Moore et al. However, two major cautions are in order: (1) There is no evidence in the present data for such a relationship, other than that the time course of the behavioral changes are compatible with the time course of the presumed anatomical rearrangements which may be taking place in the rats' brains. (2) There is, as yet, no evidence that the reinnervation seen by Raisman and Moore et al. results in functional "working" synapses. Nevertheless, in attempting to bring the present behavioral results into juxtoposition with a potential physiological mechanism, the potential for synaptic rearrangement as displayed by the adult rat brain should not be overlooked.

\section{REFERENCES}

BreMiller, R. A. A rapid technique of preparing frozen sections of small brains. Physiology and Behavior, 1971, 6, 463-464.

Dawson, R. G., Conrad, L., \& Lynch, G. Single and twostage hippocampal lesions: A similar syndrome. Experimental Neurology, 1973, 40, 263-277.

Kimble, D. P. The effects of bilateral hippocampal lesions in rats. Journal of Comparative and Physiological Psychology, 1963, 56, 273-283.

Kimble, D. P., \& Kimble, R. J. The effect of hippocampal lesions on extinction and "hypothesis" behavior in rats. Physiology and Behavior, 1970, 5, 735-738.

Lanier, L. P., \& IsaAcson, R. L. Activity changes related to the location of lesions in the hippocampus. Behavioral Biology, 1975, 13, 59-69.

Moore, R. Y., Bjorklund, A. \& Stenevi, U. Plastic changes in the adrenergic innervation of the rat septal area in response to denervation. Brain Research, 1971, 33, 13-35.

Moore, R. Y., BJorkiund, A., \& Stenevi, U. Growth and plasticity of adrenergic neurons. In F. O. Schmitt \& F. G. Worden (Eds.), The neurosciences, Third Study Program. Cambridge, Mass: M.I.T. Press, 1974. Pp. 961-977.

Raisman, G. Neuronal plasticity in the septal nuclei of the adult rat. Brain Research, 1969, 14, 25-48.

Raisman, G., \& Field, P. M. A quantitative investigation of the development of collateral reinnervation after partial deafferentation of the septal nuclei. Brain Research, 1973, 50, 241-264.

Roberts, W. W., Dember, W. N., \& Brodwick, M. Alternation and exploration in rats with hippocampal lesions. Journal of Comparative and Physiological Psychology, 1962, 55, 695-700.

(Received for publication December 17, 1975; revision received January $20,1976$. 